\title{
Severe gestational hyperthyroidism complicated by cardiac arrest - a case report
}

\author{
Alina Kuryłowicz ${ }^{1}$, Grzegorz Niewiński ${ }^{2}$, Andrzej Kański², Paweł Derlatka ${ }^{3}$, \\ Krzysztof Czajkowski ${ }^{3}$, Tomasz Bednarczuk ${ }^{1}$, Urszula Ambroziak ${ }^{1}$ \\ ${ }^{1}$ Department of Internal Medicine and Endocrinology, Medical University of Warsaw, Poland \\ ${ }^{2} 2^{\text {nd }}$ Department of Anesthesiology and Intensive Therapy, Medical University of Warsaw, Poland \\ ${ }^{3} 2^{\text {nd }}$ Department of Obstetrics and Gynecology, Medical University of Warsaw, Poland
}

Gestational hyperthyroidism (GH) is the main cause of thyrotoxicosis during the first trimester of pregnancy, diagnosed in about $1-3 \%$ of pregnancies. The course of $\mathrm{GH}$ is usually mild and self-limiting, most patients do not require treatment due to spontaneous remission of the symptoms.

We present a case of a 30-year-old patient with severe gestational hyperthyroidism, complicated by cardiac arrest despite treatment. The primipara at 8 weeks of gestation was referred to the Department of Obstetrics and Gynecology due to severe dyselectrolytemia (natrium $123 \mathrm{mmol} / \mathrm{L}$, potassium $2.34 \mathrm{mmol} / \mathrm{L}$ ) in the course of persistent vomiting. Since the early childhood the patient had been under the care of gastroenterologist due to recurrent vomiting of unknown etiology (the psychogenic causes could not be excluded). The symptoms worsened during two weeks prior to hospitalization, being accompanied by increased anxiety, hand tremor, and lost about 10 kilograms. On admission, her medical condition was severe. She presented with signs of dehydration, upper limb tremor and tachycardia 120/min. The thyroid gland was not enlarged, without audible murmur. Based on the clinical picture and markedly elevated free thyroid hormones impending hyperthyroid storm was diagnosed and thiamazole iv $120 \mathrm{mg} / 24 \mathrm{~h}$ was initiated. The patient was transferred to Intensive Care Unit. Based on anti-TSH receptors antibodies and thyroid ultrasound (both normal) the diagnosis of severe $\mathrm{GH}$ associated with hyperemesis gravidarum was established. Thiamzole along with electrolytes and parenteral nutrition was continued. On day 2, sudden cardiac arrest (SCA) occurred twice: first due to ventricular fibrillation and next due to asystole, with successful resuscitation. After SCA, the patient received parenteral hydrocortisone $300 \mathrm{mg} / 24 \mathrm{~h}$ and required a temporary infusion of pressor amines. On day 4 , in the absence of fetal heart rate on ultrasound, a surgical termination of pregnancy was performed. Her condition and thyroid hormones were gradually improving (Figure 1). Given a high

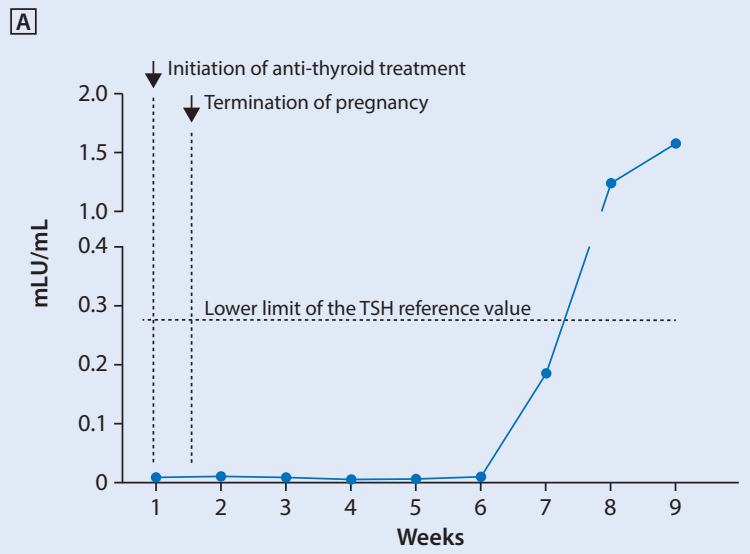

B

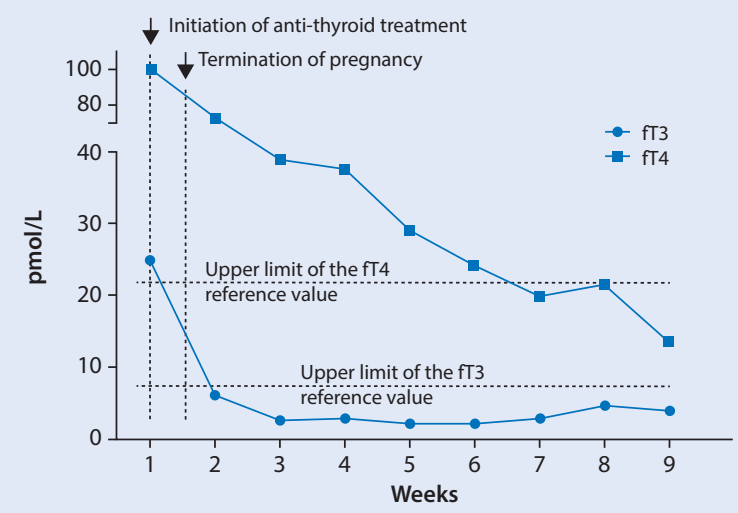

Figure 1. Changes in serum thyrotropin concentrations (A), free thyroxin and free triiodothyronine (B) during the observation period

Corresponding author:

Urszula Ambroziak

Department of Internal Medicine and Endocrinology, Medical University of Warsaw

Banacha St. 1a, 02-097 Warsaw, Poland

tel.: +48 (22) 599 2975, fax: +48 (22) 5991975

e-mail: uambroziak@wum.edu.pl 
risk of severe hyperthyroidism and the related complications during next pregnancies, despite the potentially reversible cause of SCA, the patient was deemed eligible for implantation of a cardioverter-defibrillator as a secondary prevention.

Thyroid hormones can enhance $\mathrm{Na}^{+} / \mathrm{K}^{+}$ATPase activity both, by the genomic mechanism as well as by the increase of the intracellular CAMP accumulation, resulting in potassium influx into the cells and subsequent hypokalemia. It is important to remember about metabolic alkalosis caused by intense vomiting, which could increase a shift of potassium from the plasma into the cells (via stimulation of the $\mathrm{Na}^{+} / \mathrm{H}^{+}$exchange and a subsequent activation of $\mathrm{Na}^{+} / \mathrm{K}^{+}$ ATPase activity), with a parallel rise of plasma $\mathrm{HCO}_{3}$ concentration exceeding the capacity of the renal proximal tubule to reabsorb this anion, though potassium is excreted as an obligate cation partner to the bicarbonate. Finally, severe intravascular volume depletion could also aggravate potassium lost via aldosterone-mediated mechanisms. We suspect that severe intracellular hypokalemia was the main cause of ventricular fibrillation responsible for the first SCA, even though the plasma potassium concentration was being corrected by an intravenous infusion. In turn, in the prompt re-administration of potassium via the central vein catheter we see the cause of the second SCA, which took place in the course of bradycardia and subsequent asystole. 